\title{
Prehospital Heart Rate and Blood Pressure Increase the Positive Predictive Value of the Glasgow Coma Scale for High-Mortality Traumatic Brain Injury
}

\author{
Andrew Reisner, ${ }^{1,2}$ Xiaoxiao Chen, ${ }^{1}$ Kamal Kumar, and Jaques Reifman ${ }^{1}$
}

\begin{abstract}
We hypothesized that vital signs could be used to improve the association between a trauma patient's prehospital Glasgow Coma Scale (GCS) score and his or her clinical condition. Previously, abnormally low and high blood pressures have both been associated with higher mortality for patients with traumatic brain injury (TBI). We undertook a retrospective analysis of 1384 adult prehospital trauma patients. Vital-sign data were electronically archived and analyzed. We examined the relative risk of severe head Abbreviated Injury Scale (AIS) 5-6 as a function of the GCS, systolic blood pressure (SBP), heart rate $(\mathrm{HR})$, and respiratory rate (RR). We created multi-variate logistic regression models and, using DeLong's test, compared their area under receiver operating characteristic curves (ROC AUCs) for three outcomes: head AIS 5-6, all-cause mortality, and either head AIS 5-6 or neurosurgical procedure. We found significant bimodal relationships between head AIS 5-6 versus SBP and HR, but not RR. When the GCS was <15, ROC AUCs were significantly higher for a multi-variate regression model (GCS, SBP, and HR) versus GCS alone. In particular, patients with abnormalities in all parameters (GCS, SBP, and HR) were significantly more likely to have high-mortality TBI versus those with abnormalities in GCS alone. This could be useful for mobilizing resources (e.g., neurosurgeons and operating rooms at the receiving hospital) and might enable new prehospital management protocols where therapies are selected based on TBI mortality risk.
\end{abstract}

Key words: blood pressure; Glasgow Coma Scale; heart rate; prehospital; traumatic brain injury

\section{Introduction}

T He Glasgow Coma Scale (GCS) was developed to standardize the assessment of coma and impaired consciousness after traumatic brain injury (TBI). ${ }^{1}$ As originally intended, the GCS is to be assessed only after hemodynamic resuscitation and in the absence of pharmacologic sedation, paralysis, or other forms of chemical intoxication. ${ }^{2}$ The GCS was an innovation, providing an objective method for measuring patients' global brain function. In the absence of established alternatives, use of the GCS spread to a multitude of applications outside the researchers' original intent. For example, in national guidelines for trauma patient management, a below-normal prehospital GCS is one criterion for emergency medical service (EMS) transport from the field directly to a level 1 trauma center ${ }^{3}$ and for emergency tracheal intubation after traumatic injury. ${ }^{4}$ In addition, the GCS is often relied upon in prehospital research to help control for degree of TBI (for example, see Davis and colleagues ${ }^{5}$ ), even though it was not originally intended, nor validated, for this clinical context. Indeed, the GCS is currently being used for non-TBI patients, for instance, to measure brain function in meningitis ${ }^{6}$ and hypothyroidism ${ }^{7}$ cases. Overall, the GCS has evolved to become a near-universal measure for global mental function, despite its original intent and validation in TBI patients subsequent to stabilization.

Unsurprisingly, because the GCS is applied in different ways distinct from its original intent, there is growing recognition that it may not be optimal for all these applications ${ }^{8}$ and that such widespread, inconsistent application of the GCS can cloud its interpretation. 9,10 Fundamentally, the provisos for the classic GCS - measurement after hemodynamic resuscitation and in the absence of intoxication-are incompatible with clinical decision making or research into early trauma care. Further, EMS caregivers may have less capacity for careful clinical evaluation, which may be one factor why significant discrepancies between prehospital GCS versus emergency department (ED) GCS have been reported. ${ }^{11}$ For the early stages of trauma care, it has been suggested that a simplified score, for example, either the motoronly score, or the "alert, voice, pain, unresponsive" rating, would offer reliability and convenience without much loss of clinical accuracy, because the three GCS subscales are largely redundant

${ }^{1}$ Biotechnology High Performance Computing Software Applications Institute, Telemedicine and Advanced Technology Research Center, U.S. Army Medical Research and Materiel Command, Ft. Detrick, Maryland.

${ }^{2}$ Department of Emergency Medicine, Massachusetts General Hospital, Boston, Massachusetts. 
(because of the high correlation between the three) ${ }^{8}$ However, a simplified coma score is decidedly low resolution (i.e., patients are stratified into just a small number of severity levels) and does not overcome another deficiency: mediocre outcome prediction. $^{8}$

Overall, there is an unanswered need for an accurate, practical method of assessing the severity of TBI during early trauma patient care, which could be employed in clinical decision algorithms or research investigations. A series of reports has suggested that blood pressure (BP) offers prognostic information relevant to TBI. It is intuitive that, for the initial evaluation of the trauma patient, a low GCS and low BP are correlates of mortality; ${ }^{12,13}$ the relationship between a low GCS, hypotension, and higher mortality has been quantified in classic prehospital severity scores, such as the trauma score, ${ }^{14}$ the prehospital index, ${ }^{15}$ and the "circulation, respiration, abdomen, motor, speech" (CRAMS) score. ${ }^{16}$

At the same time, hypertension is recognized as another correlate of mortality in TBI patients. In a population of TBI patients, Zafar and colleagues ${ }^{17}$ found that high and low BP were both associated with increased short-term mortality. These findings were consistent with an earlier analysis of TBI patients by Butcher and colleagues ${ }^{18}$ and another report of an association between elevated $\mathrm{BP}$ and reduced survival in trauma patients. ${ }^{19}$ Our group has previously examined how real-time computerized vital-sign analysis can enhance prehospital recognition of hemorrhagic hypovolemia, ${ }^{20,21}$ and we decided to investigate whether the findings of Zafar and colleagues ${ }^{17}$ and Butcher and colleagues ${ }^{18}$ could be diagnostically applicable to a general prehospital trauma population (i.e., patients without and with TBI). In addition to examining BP, we also sought to explore the diagnostic significance of other vital signs, such as heart rate (HR) and respiratory rate (RR), in the early evaluation of TBI, because abnormalities such as bradycardia and bradypnea are hallmarks of severe TBI. We hypothesized that it would be possible to use routine vital signs to improve the correlation between the prehospital GCS and high-mortality TBI. Accordingly, we undertook a retrospective analysis of a prehospital trauma patient database to test the hypothesis.

\section{Methods}

\section{Clinical data collection}

This was a retrospective analysis of clinical data originally collected and analyzed by Cooke and colleagues, ${ }^{22}$ with institutional review board approval, of trauma patients during transport by air ambulance from the scene of injury to a level 1 trauma center. In a convenience sample of prehospital trauma patients, vital-sign data were obtained using a Propaq 206EL monitor (Welch Allyn, Beaverton, OR) between August 2001 and April 2004 and using a PIC 50 monitor (Welch Allyn) between March 2005 and May 2007. The following data were archived using a networked personal digital assistant: electrocardiogram (ECG) and associated continuous HR; impedance pneumogram (IP) and associated continuous $\mathrm{RR}$; and systolic BP (SBP) and diastolic BP (DBP) measured intermittently at multi-minute intervals. Prospectively, prehospital GCS was assessed and documented by EMS caregivers (paramedics and critical care flight nurses) as per routine clinical operations; no focused training related to GCS assessment was provided to these EMS providers as part of this investigation. Retrospectively, clinical data for analysis were collated by chart review, including demographics, prehospital GCS, prehospital interventions, hospital treatments, coded injury descriptions (Abbreviated Injury Scale; AIS), and overall outcomes (mortality). The complete investigational data set was subsequently uploaded to our data warehousing system. ${ }^{23}$ Protected health information was not included. All subsequent data analyses were performed using MATLAB (version 7; MathWorks, Natick, MA).

\section{Vital-sign data processing}

Vital signs and other physiological data measured during prehospital clinical operations are often corrupted by artifacts. Previous research has demonstrated that automated computer algorithms can identify and remove unreliable data, leading to significant improvements in the association between vital signs and traumatic hemorrhage. ${ }^{20,24,25}$

Here, we used the same validated methodology, summarized as follows: For each vital-sign value, reliable data were identified by automated algorithms that rated each datum on an integer scale from least to most reliable. ${ }^{24,26,27} \mathrm{HR}$ and RR reliability algorithms involved analysis of ECG and IP waveforms, respectively. ${ }^{26,27}$ Briefly, when the waveforms were clean with rhythmic, consistent beats or breaths, the corresponding rates tended to be rated as reliable. Conversely, when the waveforms were noisy with irregular, heterogeneous beats or breaths, the rates were rated as unreliable. In previous validation, these algorithms' ratings of ECG and IP waveforms and reliability of the corresponding HR and RR typically concurred with the opinion of clinicians. The BP reliability algorithm determined whether the ratios between SBP, DBP, and mean pressure were physiological and whether the HR measured by the inflatable oscillometric cuff matched the ECG HR. ${ }^{24}$

For the TBI analysis, we studied the mean of the reliable HR, $\mathrm{RR}$, and SBP in the initial 15-min transportation, so each patient had no more than one HR, RR, and SBP datum. In previous research investigating the relationship between prehospital vital signs and clinically significant blood loss, we found that taking the average over 15 min was an effective measure to reduce transient variability and unreliable measurements, leading to an improved association with clinical outcomes. ${ }^{20,28}$

\section{Subject selection}

For analyses involving $\mathrm{HR}$ as an independent variable, we studied patients with an available GCS score and at least one reliable HR value in the initial $15 \mathrm{~min}$ of transportation. For analyses involving RR as an independent variable, we studied patients with an available GCS score and at least one reliable RR value in the initial $15 \mathrm{~min}$ of transportation (and further examined a subset of patients who were spontaneously breathing, i.e., nonintubated). For analyses involving SBP as an independent variable, we studied patients with an available GCS score and at least one reliable SBP value in the initial 15-min transportation. For the multi-variate analyses, we studied patients with available GCS scores and at least one reliable HR value and one reliable SBP value in the initial 15 min of transportation. We excluded the 1 patient who left against medical advice, because his injuries and outcome were unknown.

\section{Definition of high-mortality traumatic brain injury for investigation of diagnostic test characteristics}

It can be difficult to differentiate between patients who died as a result of TBI versus coexistent injuries and other clinical factors. Therefore, we investigated three parallel definitions of highmortality TBI. Our assumption was that any valid study finding should be consistent for any reasonable definition of high-mortality TBI (i.e., consistent across all three outcome definitions).

Our primary outcome definition was head AIS score of 5 or 6 . The AIS is a well-validated, widely used scoring system that assigns a score from 0 to 6 based on the anatomic injury pattern, rating how likely the patient is to die from the injury. ${ }^{29}$ The specific AIS cutoff (i.e., 5-6) for high-mortality TBI was selected post hoc after a preliminary analysis to identify the discriminatory capability of SBP, HR, and RR as a function of specific AIS scores. For that 
preliminary analysis, we calculated the relative risk of head AIS 3-6, 4-6, and 5-6 as a function of different ranges of SBP $(\mathrm{x} \geq \mathrm{SBP}>\mathrm{x}+25 \mathrm{~mm} \mathrm{Hg}), \mathrm{HR}(\mathrm{x} \geq \mathrm{HR}>\mathrm{x}+20$ beats/min $)$, and RR $(x \geq R R>x+5$ breaths/min). Relative risk was defined as the risk for patients within the range (i.e., ratio of positive cases to total cases within the range) divided by the risk for patients outside of the range (i.e., ratio of positive cases to total cases outside of the range). Confidence intervals (CIs) were computed as per Daly. ${ }^{30}$ For testing the significance of relative risks for different ranges, we compared each against the relative risks of specific reference ranges (reference ranges for SBP, 100-125 mm Hg; HR, 80-100 beats/ min; RR, 30-35 breaths/min) using the method of Altman and Bland. ${ }^{31}$ This analysis led us to define the primary outcome as head AIS 5-6.

We explored two secondary definitions of high-mortality TBI. Specifically, we examined all-cause mortality. We also examined head AIS 5-6 or documented neurosurgical procedure ("head AIS 5-6/procedure") as a secondary outcome for those cases in which a neurosurgical intervention was performed that may have prevented an otherwise fatal TBI.

\section{Diagnostic test characteristics of Glasgow Coma Scale, systolic blood pressure, and heart rate for high-mortality traumatic brain injury}

After the preliminary analysis suggested that there was no significant association between RR and TBI, RR was excluded from further analysis.

We investigated the diagnostic performance of the following:

- GCS alone as the independent variable.

- SBP and HR; to accommodate their bimodal relationship with TBI (i.e., both high and low values of SBP and HR are associated with an increased risk of TBI), we used relative risks as follows. The preliminary analysis (detailed above) yielded relative risk as a function of each SBP and HR. We fitted a cubic spline to these curves, obtaining a mathematical expression for TBI relative risk as a function of SBP or HR values. We then used the computed relative risks $\left(\mathrm{SBP}_{\text {Risk }}\right.$ and $\mathrm{HR}_{\text {Risk }}$ ) as inputs to multi-variate logistical regression models trained to predict each of the investigational outcomes.
- A multivariate logistic regression model using all three investigational predictors (GCS, $\mathrm{SBP}_{\text {Risk }}$, and $\mathrm{HR}_{\text {Risk }}$ ).

We computed receiver-operating characteristic (ROC) curves for the investigational outcomes to evaluate their diagnostic performance. We compared the areas under each ROC curve (ROC AUC) using DeLong and colleagues' method ${ }^{32}$ (significance level of $p<0.05$ ).

\section{Results}

Our data set contained 1384 subjects with at least one nonzero vital-sign datum. We identified 1289 subjects with at least one reliable HR value in the first $15 \mathrm{~min}, 649$ with at least one reliable RR value (of these, 499 were spontaneously breathing), 1247 with at least one reliable SBP value, and 1158 with at least one reliable $\mathrm{SBP}$ and one reliable HR value. Table 1 shows the characteristics of these 1247 subjects, as well as the two subpopulations (GCS $<15$ and GCS $\leq 8$ ) analyzed in the multi-variate analysis. GCS from the ED was available for $88 \%$ of the study population. Compared with prehospital GCS, average ED GCS was 0.03 points lower and the standard deviation of their differences was 2.1.

Unless otherwise specified, we used $p<0.05$ for significant results reported below.

\section{Relative risk of traumatic brain injury as a function of prehospital vital signs}

When we computed the relative risk of high-mortality TBI (i.e., head AIS 5-6, 4-6, and 3-6) as a function of prehospital SBP, we found the following:

- The relative risks given low SBP $(<100 \mathrm{~mm} \mathrm{Hg})$ were significantly different than the relative risks given SBP within the reference range for all three head AIS cutoffs (i.e., AIS 5-6, 46, and 3-6). Relative risks given SBP $<100 \mathrm{~mm} \mathrm{Hg}$ were 2.6 (95\% CI, 1.3-5.2), 2.0 (1.3-3.0), and 1.5 (1.1-2.0) for head AIS 5-6, 4-6, and 3-6, respectively. Given SBP within the reference range $(125 \mathrm{~mm} \mathrm{Hg} \geq \mathrm{SBP}>100 \mathrm{~mm} \mathrm{Hg}$ ), relative risks were 0.5 $(0.2-1.1), 0.7(0.5-1.0)$, and $0.9(0.7-1.2)$, respectively.

Table 1. Population Description for the Overall Study Population and Key Subpopulations

\begin{tabular}{|c|c|c|c|}
\hline Characteristics & Any GCS & $G C S<15$ & $G C S \leq 8$ \\
\hline Population size $(n)$ & 1,158 & 530 & 225 \\
\hline Men $(\%)$ & $836(72)$ & $374(71)$ & $162(72)$ \\
\hline Women $(\%)$ & $319(28)$ & $154(29)$ & $62(28)$ \\
\hline Mean age, years & $38(15)$ & $36(15)$ & $36(15)$ \\
\hline Blunt injury $(\%)$ & $1,012(87)$ & $478(90)$ & $191(85)$ \\
\hline Penetrating injury (\%) & $125(11)$ & $44(8)$ & $31(14)$ \\
\hline Mortality $(\%)$ & $82(7)$ & $73(14)^{\mathrm{a}}$ & $65(29)^{a}$ \\
\hline Tracheal intubation (\%) & $253(22)$ & $236(45)^{\mathrm{a}}$ & $203(90)^{\mathrm{a}}$ \\
\hline $24-\mathrm{h}$ PRBC vol $\geq 1(\%)$ & $220(19)$ & $122(23)^{\mathrm{a}}$ & $75(33)^{\mathrm{a}}$ \\
\hline 24-h PRBC vol $\geq 1$ and hemorrhagic injury $(\%)$ & $106(9)$ & $50(9)$ & $31(14)^{\mathrm{a}}$ \\
\hline 24-h PRBC vol $\geq 4(\%)$ & $109(9)$ & $64(12)^{\mathrm{a}}$ & $42(19)^{\mathrm{a}}$ \\
\hline 24-h PRBC vol $\geq 4$ and hemorrhagic injury $(\%)$ & $66(6)$ & $34(6)$ & $23(10)^{\mathrm{a}}$ \\
\hline Head AIS $3(\%)$ & $116(10)$ & $87(16)^{\mathrm{a}}$ & $49(22)^{\mathrm{a}}$ \\
\hline Head AIS $4(\%)$ & $76(7)$ & $64(12)^{\mathrm{a}}$ & $41(18)^{\mathrm{a}}$ \\
\hline Head AIS 5-6 (\%) & $41(4)$ & $40(8)^{\mathrm{a}}$ & $35(16)^{\mathrm{a}}$ \\
\hline Intracranial pressure monitoring or craniotomy $(\%)$ & $57(5)$ & $56(11)^{\mathrm{a}}$ & $45(20)^{\mathrm{a}}$ \\
\hline
\end{tabular}

"Hemorrhagic injury" was a documented laceration or fracture of a solid organ, a thoracic or abdominal hematoma, a vascular injury that required operative repair, or a limb amputation.

"Subpopulation significantly different from the "Any GCS" study population (using chi-squared test for proportion data; Student's $t$-test for mean age). AIS, abbreviated injury scale; GCS, Glasgow Coma Scale; PRBC, packed red blood cells. 
- The relative risks given high $\mathrm{SBP}(\geq 175 \mathrm{~mm} \mathrm{Hg})$ were significantly different than the relative risks given HR within the reference range for head AIS 5-6 and for AIS 4-6 (but not for AIS 3-6). Relative risks given SBP $\geq 175 \mathrm{~mm} \mathrm{Hg}$ were 3.6 (95\% CI, 1.5-8.7), $1.6(0.8-3.3)$, and $1.3(0.8-2.2)$, for head AIS 5-6, 4-6, and 3-6, respectively.

When we computed the relative risk of high-mortality TBI as a function of prehospital HR, we found the following:

- The relative risks given low HR ( $<60$ beats/min) were significantly different than the relative risks given HR within the reference range for all three head AIS cutoffs (i.e., AIS 5-6, 4-6, and 3-6). Relative risks given HR $<60$ beats/min were 5.9 (95\% CI, 2.9-12.3), 3.0 (1.8-5.1), and 2.0 (1.3-3.0) for head AIS 5-6, 4-6, and 3-6, respectively. Given HR within the reference range ( 100 beats $/ \mathrm{min} \geq \mathrm{HR}>80$ beats/ $\mathrm{min})$, relative risks were 0.7 (0.4-1.3), $0.7(0.5-1.0)$, and 0.8 (0.6-1.0), respectively.

- The relative risks given high HR ( $\geq 120$ beats/min) were not significantly different than the relative risks given HR within the reference range for head AIS 5-6, but they were significantly different for head AIS 4-6 and for AIS 3-6. Relative risks given $\mathrm{HR} \geq 120$ beats/min were 1.0 (95\% CI, 0.5-2.2), $1.2(0.8-1.9)$, and $1.2(0.9-1.6)$, for head AIS 5-6, 4-6, and 3-6, respectively.

When we computed the relative risk of high-mortality TBI as a function of prehospital RR, we found no statistically significant risks. This absence of significant findings persisted through all definitions of high-mortality TBI (head AIS 5-6, 4-6, and 3-6). Moreover, there were no significant findings related to RR for either the entire study population or the subset of patients who were spontaneously breathing (no airway intubation).

Figure 1 shows the relative risk of head AIS 5-6 as a function of different ranges of SBP, HR, and RR.

\section{Multi-variate regression models}

- Multi-variate models that included $S B P_{\text {Risk }}$ and $H R_{\text {Risk: }}$ For the models that did not include the GCS, we found that $\mathrm{HR}_{\text {Risk }}$ was a significant term in all of the nine investigated multi-variate regression models, whereas $\mathrm{SBP}_{\text {Risk }}$ was a significant term in eight of nine of the models (see Table 2).

- Multi-variate models that included GCS, SBP $P_{\text {Risk }}$ and $H R_{\text {Risk }}$ : For the models that did include GCS, we found that $\mathrm{HR}_{\text {Risk }}$ was a significant term in eight of the nine investigated multi-variate regression models, with elevated statistical significance $(p<0.01)$ in the three models for head AIS 5-6/procedure. $\mathrm{SBP}_{\text {Risk }}$ was a significant term in six of the nine multivariate models (head AIS 5-6 and all-cause mortality), but not in the three models for head AIS 5-6/procedure (see Table 2).

\section{Diagnostic test characteristics of the Glasgow Coma Scale alone versus multi-variate regression models}

- For the overall study population $(n=1158)$ : GCS provided rather good ROC AUCs, and the multi-variate models, including GCS, $\mathrm{SBP}_{\text {Risk}}$, and $\mathrm{HR}_{\text {Risk, }}$, did not offer a significant increase in ROC AUCs versus the GCS used alone (see Table 2).

- For patients with GCS <15: The GCS alone was less discriminatory, that is, it yielded lower ROC AUCs. The models that included the GCS, $\mathrm{SBP}_{\text {Risk }}$, and $\mathrm{HR}_{\text {Risk }}$ offered significant improvements over the GCS for all three definitions of high-mortality TBI: head AIS 5-6 (ROC AUC + 0.04); allcause mortality (ROC AUC +0.03); and head AIS 5-6/ procedure (ROC AUC +0.03; see Table 2).

- For patients with GCS $\leq 8$ : In this subpopulation, the GCS alone was less discriminatory for high-mortality TBI. The models that included the GCS, $\mathrm{SBP}_{\text {Risk }}$, and $\mathrm{HR}_{\text {Risk }}$ offered significant improvements over the GCS for all three outcomes, head AIS 5-6 (ROC AUC +0.12), mortality (ROC AUC + 0.09), and AIS 5-6/procedure (ROC AUC + 0.07; see Table 2).

\section{Example of improved risk stratification using the Glasgow Coma Scale, heart rate, and systolic blood pressure versus the Glasgow Coma Scale alone}

Figure 2 shows another distinction between the prehospital GCS alone and the multi-variate model.

- For GCS, its positive predictive value (PPV) for high-mortality TBI gradually increased as the GCS score grew more abnormal.

- The multi-variate regression model was different from the GCS (see Fig. 2). Like the GCS, the PPV for the multivariate model rose gradually as the model output was more abnormal. Unlike the GCS, there was an apparent threshold above which the PPV rose quite steeply and above which it demonstrated significantly higher PPV for high-mortality TBI, as compared with the GCS alone. This implies that patients with a combination of an abnormal GCS, abnormal BP (too high or too low), and abnormal HR (too high or too low) had a $>50 \%$ probability of high-mortality TBI.

\section{Discussion}

In this report, we investigated whether vital signs could be used to improve prehospital GCS as a diagnostic indicator of highmortality TBI. Improved prehospital identification of highmortality TBI could be valuable, guiding prehospital protocols and mobilizing resources at the receiving facility in an efficient manner. It could also offer an improved tool for research.

This investigation built on previous reports observing a distinct bimodal relationship between BP and clinical outcomes in TBI patient populations. ${ }^{17,18}$ Consistent with those earlier reports, we identified a bimodal relationship between prehospital BP and the study outcomes as well as several novel findings:

- We identified a bimodal relationship between high-mortality TBI and prehospital HR.

- We found that the GCS, as documented by the EMS caregivers, did not provide additional risk stratification once the GCS was $\leq 8$ (in other words, all patients with GCS $\leq 8$ had similar rates of high-mortality TBI; see Table 2).

- By combining the GCS and prehospital vital signs in a multivariate model, after accounting for the bimodal relationships, it was possible to improve the identification of the highest-mortality TBI. For example, it was possible to identify an extremely high-risk subgroup that evidenced $>50 \%$ probability of all-cause mortality. In contrast, the lowest GCS did not offer such positive predictive value (see Fig. 2). Of course, a clinical score involving relative risk calculation plus multi-variate regression is not feasible for a bedside clinician, but it is well within the capabilities of emerging information technologies (for examples, see previous reports ${ }^{20,21}$ ). 

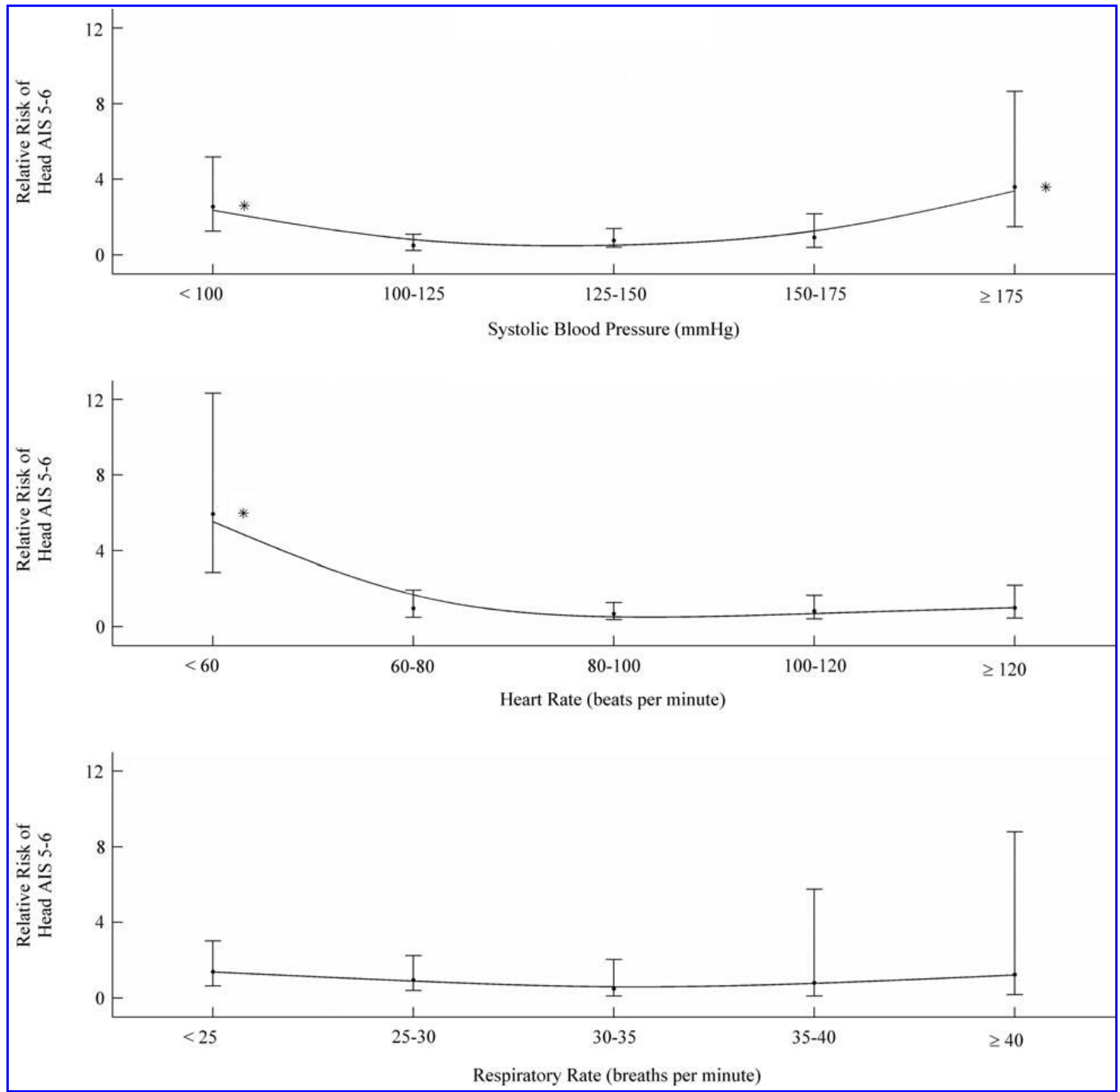

FIG. 1. Prehospital vital signs versus relative risk of head abbreviated injury scale (AIS) 5-6. Error bars signify $95 \%$ confidence interval. Solid line indicates the cubic spline fit to data. *Statistically significant difference $(p<0.05)$ from the baseline reference range (reference ranges: systolic blood pressure, $100-125 \mathrm{~mm} \mathrm{Hg}$; heart rate, $80-100$ beats/min; respiratory rate, 30-35 breaths/min).

\section{Hemodynamics of high-mortality traumatic brain injury: examining the bimodal relationships}

What was the basis of the bimodal relationship between hemodynamics and high-mortality TBI? High BP and low HR are hallmarks of the Cushing reflex, a well-known hemodynamic response to elevated intracranial pressure. The association between low BP and high HR in high-mortality TBI is not as clear. Major mechanism polytrauma is likely a root cause, which can result in hemorrhagic hypovolemia (and hypotension and tachycardia) as well as high-mortality TBI. This is suggested by Table 1 , where it is apparent that the subpopulation with lower GCS had higher rates of blood transfusion coincident with explicitly hemorrhagic injuries. In addition, in a few cases, the low BP could be a correlate of spinal shock. Finally, the association between mor- tality and hypotension may be causal, in that low BP causes secondary harm to the injured brain. Overall, it seems likely that the basis of the hypotension and/or tachycardia relationship with TBI is multi-factorial.

We hypothesized that there would be an association between RR and TBI. However, we did not identify any significant relationships involving RR. Possibly, the patients in this data set who had respiratory depression tended to receive early tracheal intubation, and so their RR was under control of the caregivers, not the patient's own depressed respiratory drive.

We found it necessary to consider the bimodal relationships between TBI risk versus SBP and HR. When we first performed a routine regression analysis on TBI versus $\mathrm{BP}$ and $\mathrm{HR}$, without accounting for the bimodal relationship, we did not find linear correlations because the TBI cases with abnormally high values 
Table 2. Comparison of Areas Under Receiver-Operating Characteristic Curves of the Investigative Parameters

\begin{tabular}{|c|c|c|c|}
\hline \multirow[b]{2}{*}{ Investigated variables } & \multicolumn{3}{|c|}{ ROC AUC $(95 \% C I)$} \\
\hline & $\begin{array}{c}\text { Any GCS } \\
\text { Total }=1,158 \text { subjects }\end{array}$ & $\begin{array}{c}G C S<15 \\
\text { Total }=530 \text { subjects }\end{array}$ & $\begin{array}{c}G C S \leq 8 \\
\text { Total }=225 \text { subjects }\end{array}$ \\
\hline $\begin{array}{l}\text { Head AIS 5-6 } \\
\text { GCS } \\
\text { SBP }_{\text {Risk }}, \mathrm{HR}_{\text {Risk }} \\
\text { GCS, SBP } \text { Risk }_{\text {RR }}, \mathrm{HR}_{\text {Risk }}\end{array}$ & $\begin{array}{c}\text { Cases }=41, \text { Controls }=1,117 \\
0.90(0.86-0.93) \\
0.64(0.54-0.73)^{\mathrm{a}, \mathrm{b}, \mathrm{c}} \\
0.91(0.85-0.94)^{\mathrm{a}, \mathrm{b}}\end{array}$ & $\begin{array}{c}\text { Cases }=40, \text { Controls }=490 \\
0.80(0.76-0.85) \\
0.68(0.58-0.76)^{\mathrm{a}, \mathrm{b}, \mathrm{c}} \\
0.84(0.78-0.89)^{\mathrm{a}, \mathrm{b}, \mathrm{c}}\end{array}$ & $\begin{array}{c}\text { Cases }=35, \text { Controls }=190 \\
0.59(0.52-0.66) \\
0.65(0.54-0.74)^{\mathrm{a}, \mathrm{b}} \\
0.71(0.61-0.79)^{\mathrm{a}, \mathrm{b}, \mathrm{c}}\end{array}$ \\
\hline $\begin{array}{l}\text { All-cause mortality } \\
\text { GCS } \\
\text { SBP }_{\text {Risk }}, \mathrm{HR}_{\text {Risk }} \\
\text { GCS, } \mathrm{SBP}_{\text {Risk }}, \mathrm{HR}_{\text {Risk }}\end{array}$ & $\begin{array}{c}\text { Cases }=82, \text { Controls }=1,076 \\
0.85(0.80-0.90) \\
0.68(0.61-0.74)^{\mathrm{a}, \mathrm{b}, \mathrm{c}} \\
0.88(0.83-0.91)^{\mathrm{a}, \mathrm{c}}\end{array}$ & $\begin{array}{c}\text { Cases }=73, \text { Controls }=457 \\
0.82(0.77-0.86) \\
0.66(0.59-0.73)^{\mathrm{a}, \mathrm{b}, \mathrm{c}} \\
0.85(0.80-0.89)^{\mathrm{a}, \mathrm{b}, \mathrm{c}}\end{array}$ & $\begin{array}{c}\text { Cases }=65, \text { Controls }=160 \\
0.65(0.59-0.70) \\
0.66(0.58-0.74)^{\mathrm{a}, \mathrm{b}} \\
0.74(0.66-0.81)^{\mathrm{a}, \mathrm{b}, \mathrm{c}}\end{array}$ \\
\hline $\begin{array}{l}\text { Head AIS 5-6/procedure } \\
\text { GCS } \\
\text { SBP }_{\text {Risk }}, \mathrm{HR}_{\text {Risk }} \\
\text { GCS, SBP } \text { Risk }_{\text {Risk }}, \mathrm{HR}_{\text {Ris }}\end{array}$ & $\begin{array}{c}\text { Cases }=83, \text { Controls }=1,075 \\
0.89(0.86-0.92) \\
0.62(0.55-0.68)^{\mathrm{a}, \mathrm{b}, \mathrm{c}} \\
0.90(0.86-0.93)^{\mathrm{b}}\end{array}$ & $\begin{array}{c}\text { Cases }=81, \text { Controls }=449 \\
0.78(0.73-0.82) \\
0.63(0.56-0.69)^{\mathrm{a}, \mathrm{b}, \mathrm{c}} \\
0.81(0.77-0.86)^{\mathrm{b}, \mathrm{c}}\end{array}$ & $\begin{array}{c}\text { Cases }=68, \text { Controls }=157 \\
0.55(0.49-0.62) \\
0.59(0.50-0.67)^{\mathrm{b}} \\
0.62(0.54-0.70)^{\mathrm{b}}\end{array}$ \\
\hline
\end{tabular}

Shown are comparisons of areas under receiver operating characteristic curves (ROC AUCs) of the following investigative variables: 1) GCS; 2) the multi-variate regression model using the relative risk of traumatic brain injury computed from SBP ( $\left.\mathrm{SBP}_{\text {Risk }}\right)$ and from $\mathrm{HR}$ ( $\left.\mathrm{HR}_{\text {Risk }}\right)$; and 3$)$ the multivariate regression model using GCS, $\mathrm{SBP}_{\text {Risk }}$, and $\mathrm{HR}_{\text {Risk }}$.

${ }^{a} \mathrm{SBP}_{\text {Risk }}$ term was statistically significant in the multi-variate regression model.

${ }^{\mathrm{b}} \mathrm{HR}$ Risk

${ }^{c}$ ROC AUC was statistically significantly different from that of GCS.

AIS, abbreviated injury scale; CI, confidence interval; GCS, Glasgow Coma Scale; ROC, receiver operating characteristic; AUC, area under the curve; SBP, systolic blood pressure; HR, heart rate.

cancelled out TBI cases with abnormally low values. Interestingly, most of the classic prehospital severity scores (e.g., the trauma score,${ }^{14}$ the prehospital index, ${ }^{15}$ the CRAMS score, ${ }^{16}$ and the newer Glasgow Coma Scale, Age, and Systolic Blood Pressure ${ }^{13}$ / Mechanism, Glasgow Coma Scale, Age, and Arterial Pressure scores ${ }^{12}$ ) do not account for the prognostic value of hypertension and bradycardia in patients with TBI. We speculate that superior overall severity scores could be developed by accounting for the bimodal relationship between hemodynamics and high-mortality TBI.

\section{Clinical implications}

Although originally intended for use after initial resuscitation, the GCS has been adopted for the earliest stages of trauma care, although it is not optimal for that context (see Introduction). Indeed, in this study, we found that below a cutoff of 8 , the prehospital GCS offered no additional discriminatory value (see ROC AUCs in Table 2). Given the lowest level of prehospital GCS (=3), the likelihood of mortality was $37 \%$ (see Fig. 2). In contrast, using prehospital HR and SBP with the GCS, it was possible to further risk stratify patients with a prehospital GCS $\leq 8$. For example, given those patients with a low GCS and abnormal HR and SBP, we found a probability (>50\%) of mortality risk (see Fig. 2).

How could a superior tool for early estimation of TBI mortality risk be useful? First, this measure could be applied in research to control for TBI severity in biostatistical analyses of prehospital care (for instance, studies such as Davis and colleagues ${ }^{5}$ ). Second, such a tool may be useful for triage applications, such as determining which patients should receive priority care or involvement of a neurosurgeon at the earliest juncture. Third, improved risk stratification could be used to develop more rational clinical decision-making algorithms for prehospital management. For example, transport speed might be the highest priority (e.g., no delay for tracheal intubation) for patients with the highest TBI risk, in case the patient requires immediate decompressive neurosurgery, and osmotherapy may be useful, whereas permissive hypoten$\operatorname{sion}^{33}$ would be contraindicated. In contrast, for patients with depressed consciousness who are unlikely to have high-mortality TBI based on the patterns of GCS, HR, and SBP, it may prove judicious to delay transportation long enough to secure the airway and protect against aspiration, and permissive hypotension could offer more benefit than risk. In summary, based on a patient's quantitative risk of high-mortality TBI, different prehospital interventions may offer different risk-benefit profiles. Further research is warranted into novel prehospital protocols in which decision making is dependent on the quantitative risk of lifethreatening TBI.

As a practical matter, the multi-variate analysis used in this investigation requires computer analysis; such a tool is well within today's in- and prehospital capabilities. Indeed, our research team has currently deployed such automated computational devices, networked to a Propaq 206 patient monitor (Welch Allyn), on board Boston Medflight helicopters for prospective trials of advanced decision-support algorithms. ${ }^{21}$ In practice, after the GCS score was electronically documented by a caregiver, the informatics system could process the patient's recent BP and HR measurements, automatically identify and exclude any unreliable vital-sign values, compute $\mathrm{HR}_{\text {Risk }}$ and $\mathrm{SBP}_{\text {Risk }}$, and output the multi-variate regression model result.

\section{Limitations}

In a few of the multi-variate models, either the $\mathrm{SBP}_{\text {Risk }}$ term or the $\mathrm{HR}_{\text {Risk }}$ term did not reach statistical significance. However, we do not consider each and every outcome as a distinct hypothesis. Rather, we are testing the overall hypothesis that information from $\mathrm{SBP}_{\text {Risk }}$ and $\mathrm{HR}_{\text {Risk }}$ can significantly improve on the ability of the GCS to identify patients with high-mortality TBI. The results shown in Table 2 demonstrate a consistent pattern supporting this hypothesis in patients with a GCS $<15$. In contrast, GCS $=15$ was 


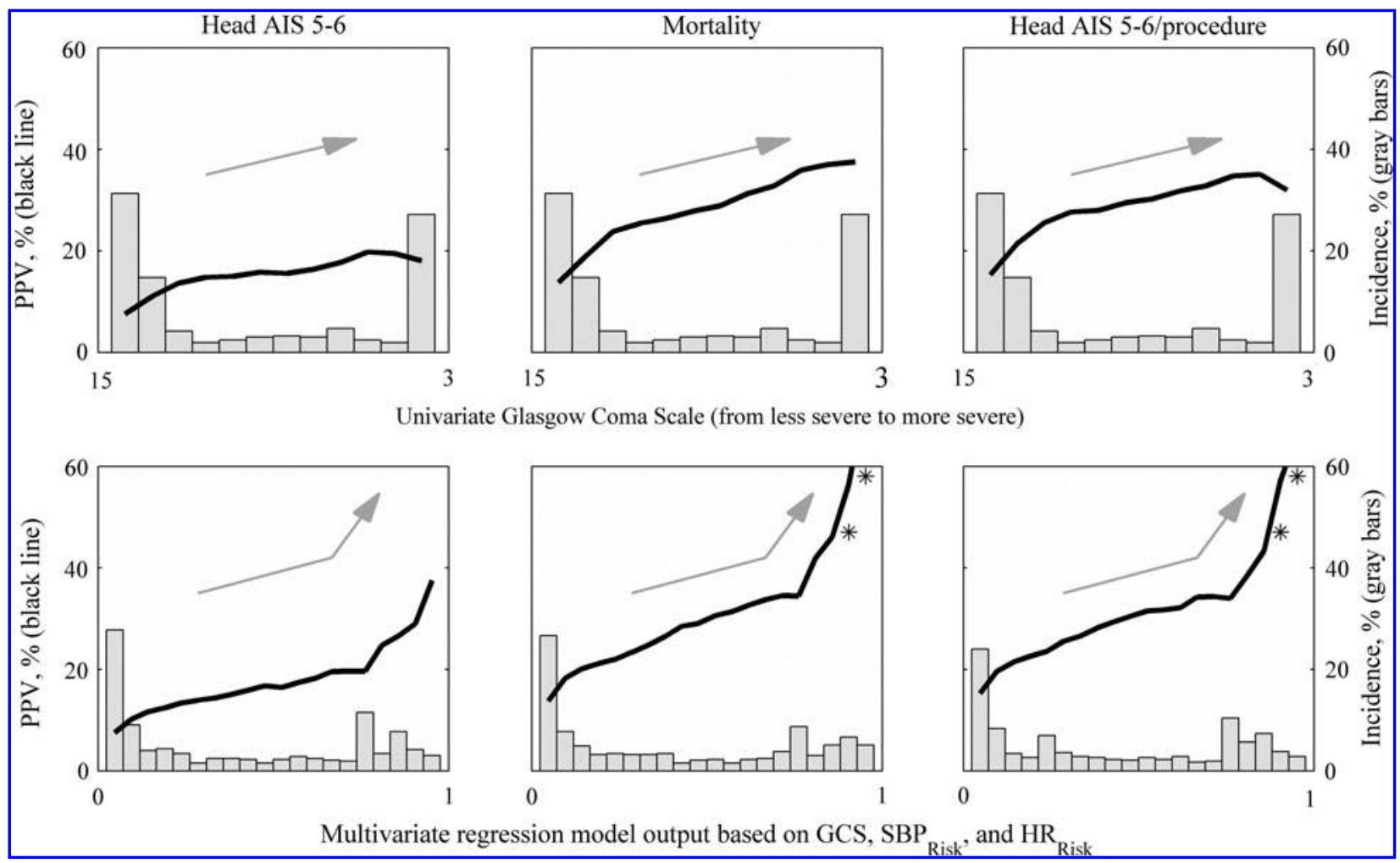

FIG. 2. Positive predictive value (PPV) of Glasgow Coma Scale (GCS) versus PPV of multi-variate regression model. Compared with GCS alone (top row), the multi-variate regression model (bottom row) better identified a subset of patients at elevated risk of highmortality traumatic brain injury (TBI), either defined by head AIS 5-6 (left column), all-cause mortality (middle column), or head AIS 5-6/procedure (right column). Solid black lines show the PPV of high-mortality TBI for patients $\leq$ GCS score (top row) or $\geq$ multivariate model output (bottom row). Distribution of patients with each GCS score is also shown (shaded bars, top row) as is the distribution of patients with different ranges of regression model outputs (shaded bars, bottom row). *Multi-variate classifier's PPV was significantly greater than PPV of GCS $=3\left(p<0.05\right.$, chi-square test). $\mathrm{SBP}_{\text {Risk }}$, systolic blood pressure relative risk; $\mathrm{HR}_{\mathrm{Risk}}$, heart rate relative risk.

very effective at identifying the majority of patients who had a very low risk of high-mortality TBI; the associated ROC AUC was quite high, without much room for improvement.

Second, this analysis focused on early identification of highmortality TBI, because optimizing survival is a primary goal of prehospital and early hospital care. However, preventing disability is as important to consider as survival. Whether BP and HR offer prognostic information about functional neurological outcome was not addressed in this analysis, and future investigation into this important question is warranted.

Third, this analysis focused on the GCS measured by EMS caregivers. Our findings may not extend to the classic GCS carefully measured in-hospital after patient stabilization and the elimination of intoxicants, which are often different from prehospital measurements. ${ }^{9,10}$ Prehospital conditions are more demanding, whereas staffing is often limited to a couple of caregivers, so nuanced GCS scoring is unlikely to be a high priority: The field medic will probably not heed the difference between flexion versus extension motor responses when it is obvious that the patient has timesensitive injuries.

The final limitation relates to the precise numerical results of our analysis. For some HR and SBP ranges, there were not enough cases for a tight estimation of the associated relative risk. A larger data set would presumably yield a more accurate quantification of the HR and SBP bimodal relationship and the optimal coefficients for the multi-variate model. All the same, our findings were qual- itatively consistent with findings from other reports. ${ }^{17,18}$ We suggest that the overall findings of this report are likely valid.

\section{Conclusion}

We found that the prehospital GCS alone was unable to effectively distinguish between trauma patients with moderate risk versus the highest risk of high-mortality TBI. A multi-variate regression model with three terms-GCS, SBP, and HR-offered significantly improved test performance after accounting for the bimodal relationships between TBI versus SBP and HR. This score could be useful for guiding operations at the receiving hospital (e.g., early consultation by a neurosurgeon and readying an operating room). Further, we speculate that improved methods for the prehospital assessment of TBI risk could facilitate new prehospital management practices.

\section{Acknowledgments}

This work was supported by the Defense Health Program and the Combat Casualty Care Research Area Directorate of the U.S. Army Medical Research and Materiel Command (Fort Detrick, MD). The authors thank Christopher Jamieson for his support in formatting the manuscript and Kathleen McGuire Gilbert for editing the manuscript.

The opinions and assertions contained herein are the private views of the authors and are not to be construed as official or as 
reflecting the views of the U.S. Army or of the U.S. Department of Defense. This article has been approved for public release with unlimited distribution.

\section{Author Disclosure Statement}

No competing financial interests exist.

\section{References}

1. Teasdale, G., and Jennett, B. (1974). Assessment of coma and impaired consciousness. A practical scale. Lancet 2, 81-84.

2. The Brain Trauma Foundation. The American Association of Neurological Surgeons. The Joint Section on Neurotrauma and Critical Care. (2000). Glasgow Coma Scale score. J. Neurotrauma 17, 563-571.

3. Sasser, S.M., Hunt, R.C., Faul, M., Sugerman, D., Pearson, W.S., Dulski, T., Wald, M.M., Jurkovich, G.J., Newgard, C.D., and Lerner, E.B.; Centers for Disease Control and Prevention. (2012). Guidelines for field triage of injured patients: recommendations of the National Expert Panel on Field Triage, 2011. MMWR Recomm. Rep. 61, 1-20.

4. Dunham, C.M., Barraco, R.D., Clark, D.E., Daley, B.J., Davis, F.E., 3rd, Gibbs, M.A., Knuth, T., Letarte, P.B., Luchette, F.A., Omert, L., Weireter, L.J., and Wiles, C.E., 3rd; EAST Practice Management Guidelines Work Group. (2003). Guidelines for emergency tracheal intubation immediately after traumatic injury. J. Trauma 55, 162-179.

5. Davis, D.P., Peay, J., Sise, M.J., Vilke, G.M., Kennedy, F., Eastman, A.B., Velky, T., and Hoyt, D.B. (2005). The impact of prehospital endotracheal intubation on outcome in moderate to severe traumatic brain injury. J. Trauma 58, 933-939.

6. Magazzini, S., Nazerian, P., Vanni, S., Paladini, B., Pepe, G., Casanova, B., Crugnola, C., and Grifoni, S. (2012). Clinical picture of meningitis in the adult patient and its relationship with age. Intern. Emerg. Med. 7, 359-364.

7. Mathew, V., Misgar, R.A., Ghosh, S., Mukhopadhyay, P., Roychowdhury, P., Pandit, K., Mukhopadhyay, S., and Chowdhury, S (2011). Myxedema coma: a new look into an old crisis. J. Thyroid Res. 2011, 493462.

8. Green, S.M. (2011). Cheerio, laddie! Bidding farewell to the Glasgow Coma Scale. Ann. Emerg. Med. 58, 427-430.

9. Zuercher, M., Ummenhofer, W., Baltussen, A., and Walder, B. (2009) The use of Glasgow Coma Scale in injury assessment: a critical review. Brain Inj. 23, 371-384.

10. Marion, D.W., and Carlier, P.M. (1994). Problems with initial Glasgow Coma Scale assessment caused by prehospital treatment of patients with head injuries: results of a national survey. J. Trauma 36, 89-95.

11. Kerby, J.D., MacLennan, P.A., Burton, J.N., McGwin, G., Jr., and Rue, L.W., 3rd. (2007). Agreement between prehospital and emergency department Glasgow Coma scores. J. Trauma 63, 1026-1031.

12. Sartorius, D., Le Manach, Y., David, J.S., Rancurel, E., Smail, N., Thicoipe, M., Wiel, E., Ricard-Hibon, A., Berthier, F., Gueugniaud, P.Y., and Riou, B. (2010). Mechanism, Glasgow Coma Scale, age, and arterial pressure (MGAP): a new simple prehospital triage score to predict mortality in trauma patients. Crit. Care Med. 38, 831-837.

13. Kondo, Y., Abe, T., Kohshi, K., Tokuda, Y., Cook, E.F., and Kukita, I. (2011). Revised trauma scoring system to predict in-hospital mortality in the emergency department: Glasgow Coma Scale, age, and systolic blood pressure score. Crit. Care 15, R191.

14. Champion, H.R., Sacco, W.J., Copes, W.S., Gann, D.S., Gennarelli, T.A., and Flanagan, M.E. (1989). A revision of the trauma score. J. Trauma 29, 623-629.

15. Koehler, J.J., Baer, L.J., Malafa, S.A., Meindertsma, M.S., Navitskas, N.R., and Huizenga, J.E. (1986). Prehospital Index: a scoring system for field triage of trauma victims. Ann. Emerg. Med. 15, 178-182.

16. Gormican, S.P. (1982). CRAMS scale: field triage of trauma victims. Ann. Emerg. Med. 11, 132-135.

17. Zafar, S.N., Millham, F.H., Chang, Y., Fikry, K., Alam, H.B., King, D.R., Velmahos, G.C., and de Moya, M.A. (2011). Presenting blood pressure in traumatic brain injury: a bimodal distribution of death. J. Trauma 71, 1179-1184.

18. Butcher, I., Maas, A.I., Lu, J., Marmarou, A., Murray, G.D., Mushkudiani, N.A., McHugh, G.S., and Steyerberg, E.W. (2007). Prognostic value of admission blood pressure in traumatic brain injury: results from the IMPACT study. J. Neurotrauma 24, 294-302.

19. Ley, E.J., Singer, M.B., Clond, M.A., Gangi, A., Mirocha, J., Bukur, M., Brown, C.V., and Salim, A. (2011). Elevated admission systolic blood pressure after blunt trauma predicts delayed pneumonia and mortality. J. Trauma 71, 1689-1693.

20. Reisner, A.T., Chen, L., and Reifman, J. (2012). The association between vital signs and major hemorrhagic injury is significantly improved after controlling for sources of measurement variability. J. Crit. Care 27, 533.e1-533.e10.

21. Reisner, A.T., Khitrov, M.Y., Chen, L., Blood, A., Wilkins, K., Doyle, W., Wilcox, S., Denison, T., and Reifman, J. (2013). Development and validation of a portable platform for deploying decision-support algorithms in prehospital settings. Appl. Clin. Inform. 4, 392-402.

22. Cooke, W.H., Salinas, J., Convertino, V.A., Ludwig, D.A., Hinds, D., Duke, J.H., Moore, F.A., and Holcomb, J.B. (2006). Heart rate variability and its association with mortality in prehospital trauma patients. J. Trauma 60, 363-370.

23. McKenna, T.M., Bawa, G., Kumar, K., and Reifman, J. (2007). The physiology analysis system: an integrated approach for warehousing, management and analysis of time-series physiology data. Comput. Methods Programs Biomed. 86, 62-72.

24. Reisner, A.T., Chen, L., McKenna, T.M., and Reifman, J. (2008) Automatically-computed prehospital severity scores are equivalent to scores based on medic documentation. J. Trauma 65, 915-923.

25. Hug, C.W., Clifford, G.D., and Reisner, A.T. (2011). Clinician blood pressure documentation of stable intensive care patients: an intelligent archiving agent has a higher association with future hypotension. $\underline{\text { Crit. }}$ Care Med. 39, 1006-1014.

26. Chen, L., McKenna, T., Reisner, A., and Reifman, J. (2006). Algorithms to qualify respiratory data collected during the transport of trauma patients. Physiol. Meas. 27, 797-816.

27. Yu, C., Liu, Z., McKenna, T., Reisner, A.T., and Reifman, J. (2006). A method for automatic identification of reliable heart rates calculated from ECG and PPG waveforms. J. Am. Med. Inform. Assoc. 13, 309-320.

28. Chen, L., Reisner, A.T., Gribok, A., and Reifman, J. (2009). Exploration of prehospital vital sign trends for the prediction of trauma outcomes. Prehosp. Emerg. Care 13, 286-294.

29. Garthe, E., States, J.D., and Mango, N.K. (1999). Abbreviated injury scale unification: the case for a unified injury system for global use. Trauma 47, 309-323.

30. Daly, L.E. (1998). Confidence limits made easy: interval estimation using a substitution method. Am. J. Epidemiol. 147, 783-790.

31. Altman, D.G., and Bland, J.M. (2003). Interaction revisited: the difference between two estimates. BMJ 326, 219.

32. DeLong, E.R., DeLong, D.M., and Clarke-Pearson, D.L. (1988). Comparing the areas under two or more correlated receiver operating characteristic curves: a nonparametric approach. Biometrics 44, 837-845.

33. Kobayashi, L., Costantini, T.W., and Coimbra, R. (2012). Hypovolemic shock resuscitation. Surg. Clin. North. Am. 92, 1403-1423.

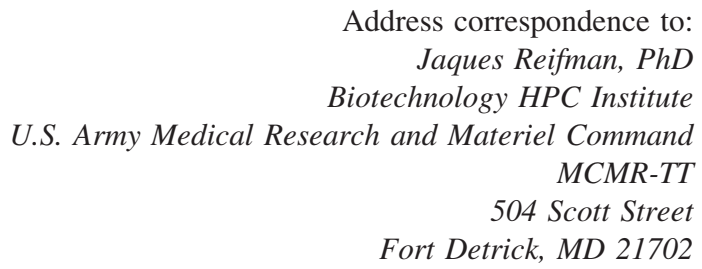

E-mail: jaques.reifman.civ@mail.mil 